J. Clin. Chem. Clin. Biochem.

Vol. 26, 1988, pp. 29-33

(C) 1988 Walter de Gruyter \& Co. Berlin - New York

\title{
Effects of Ageing on Preserved Red Blood Cell Populations as Measured by Light Scattering
}

\author{
By P. C. Bartels and A. F. M. Roijers \\ Department of Clinical Chemistry and Haematology, Medical Center Alkmaar, The Netherlands
}

(Received June 9/November 26, 1987)

Summary: Instability of reference materials may cause problems in interpreting results for assurance of quality in haemocytometry. The stability with time for a certain preserved blood specimen is also highly dependent on the principle of measurement.

In this paper some effects of ageing on red cell populations in commercially available (Ortho) Blood Controls are evaluated. Red blood cell size histograms become narrower while mean corpuscular volumes obviously decrease with time when measurements are based on light scattering intensity.

In contrast, monodisperse polystyrene spheres of different sizes show perfect stability at storage. Such artificially prepared controls should be applied to achieve optimal setting and calibration of haematology analysers.

\section{Introduction}

Multicomponent reference materials can be used for quality control purposes on automated haematology analysers (1). The daily use of commercially available preserved human blood cell specimens provides quality control data for detecting errors due to instrument malfunction and/or poor calibration. The disadvantage of nonartificial blood reference samples is the lack of long term stability (2). Red cell populations in blood controls show slight shifts due to ageing. For optimal preservation and stabilization human blood cells are suspended in a medium containing the essential nutrients for the red cells (3). After pretreatment their physical properties still closely resemble those of native red blood cells. The specifications for expiration intervals of commercially available blood specimens may depend strongly on the measurement principle. Compared with aperture impedance measurements, results obtained by use of light scattering intensity are more sensitive to haemolysis (4). In this paper some effects of ageing on red cell populations in Ortho Quality Control preserved blood specimens are evaluated during a period of 7 weeks. Measurements are performed on a ELT 800 Hematology Ana- lyzer based on light scattering intensity. Special attention is paid to gradual shifts with time which occur in the red cell size histograms.

\section{Materials and Methods}

Ortho Diagnostic Systems, B 2340 Beerse, Belgium are one of the commercial manufacturers of standardized blood preparations for quality control. The source material is of human origin. Because of their instability the white cells are removed by physical methods and replaced by fixed human red cells. Human platelets are substituted by fixed porcine platelets. According to the specifications of the manufacturer the preservation medium contains essential nutrients for maintaining the metabolism in the red blood cell. For preservation purposes neomycin sulphate, chloramphenicol and hydrocortisone are added. According to the manufacturer's instructions a batch of blood controls is stable for 7 weeks at $2-8^{\circ} \mathrm{C}$.

Ortho Hematology Blood Control specimens permit measurement of 8 haemocytometric values:

white blood cell concentration, red blood cell concentration, haemoglobin concentration, haematocrit, mean corpuscular volume, mean corpuscular haemoglobin, mean corpuscular haemoglobin concentration and platelet concentration. 
Before use Blood Control samples are allowed to reach room temperature. After resuspension of the cells by homogenizing for 5 minutes on a roller mixer a sample is run on the ELT 800. Optimal performance of the analyser is assured by adherence to the various maintenance and control procedures as specified by the manufacturer (5).

Monodisperse polystyrene spheres (Dynospheres ${ }^{\circledR}$ ), Dyno Industrier A. S., 2001 Lillestrom, Norway were applied for primary setting of the haematology analyser.

The instrument cannot measure automatically the absolute distribution width of a red blood cell size histogram at half maximum; the distribution width at half maximum is therefore manually estimated from the original red blood cell size histogram. This value is converted into femtoliters by utilizing a calibration factor. The $\mathrm{X}$-axis (voltage channel numbers) was calibrated by measuring the distance between two peaks of red blood cell size histograms with known and markedly different mean corpuscular volume values.

\section{Subjects}

Two patients with iron depletion and vitamin $B_{12}$ deficiency respectively are selected to demonstrate the characteristics of red cell size histograms.

\section{Results}

Examples of histograms generated for each of the 3 different suspensions of monodisperse polystyrene spheres are shown in figure 1. Each population of spheres gives rise to a very narrow histogram. The voltage channel numbers in the histogram corresponding with the peak values cover a relatively wide range. The positions of the respective peaks are used for establishing optimal adjustment of the haematology analyser.

Haemocytometric results were evaluated for 5 different batches of "Abnormal" as well as "Normal" Ortho Blood Controls during a period of 7 weeks. A typical example of changes occurring in a preserved red blood cell population is depicted in figure 2 . The peak volt-

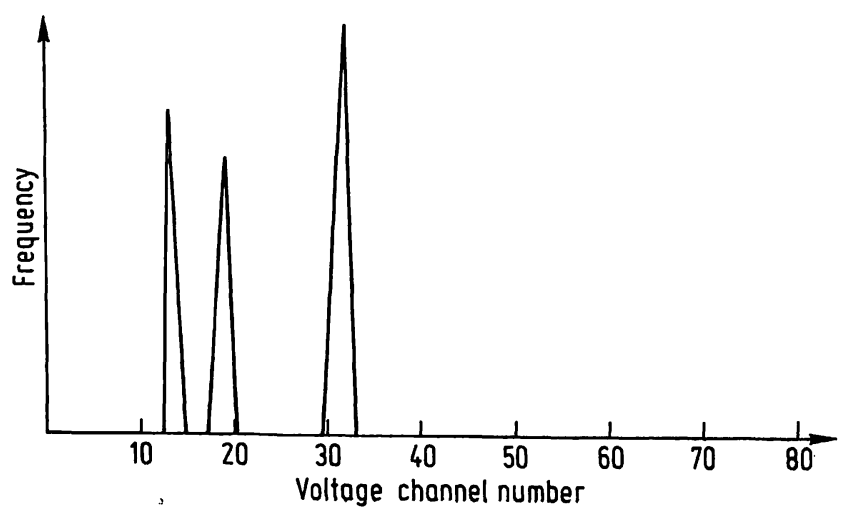

Fig. 1. Histogram obtained from an ELT 800 for several batches of regular grade polystyrene spheres. From the left to the right in the figure the peaks belong to Dynospheres ${ }^{\circledR}$ with lot numbers Q435, Q402, Q443 with diameters of respectively 5.5, 6.4 and $10.1 \mu \mathrm{m}$.

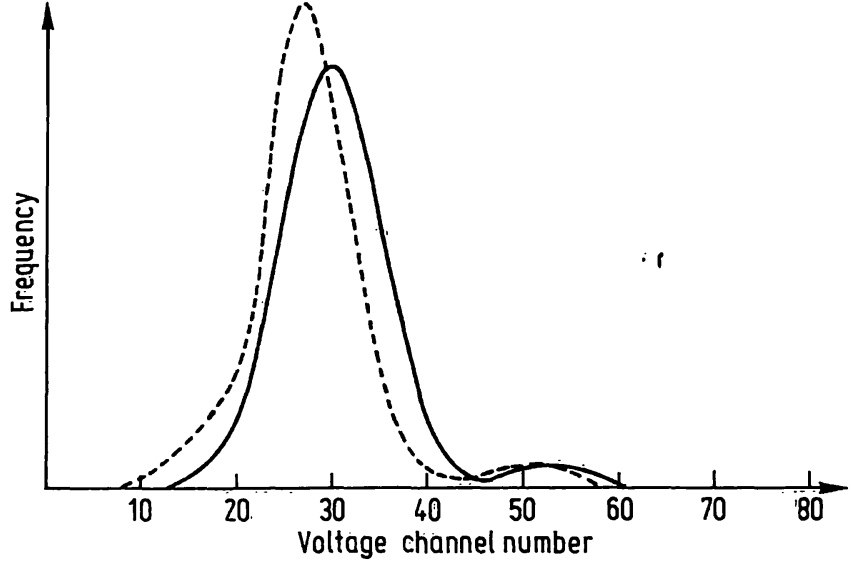

Fig. 2. Typical effects of ageing on red cell populations in a preserved Ortho Abnormal Blood Control specimen. The histogram at the right $(-)$ is derived from a sample immediately after receipt. The histogram on the left $(---)$ is obtained from a sample of the same batch 6 weeks later.

age channel numbers for all batches of preserved Ortho blood specimens also show a shift to the left. In most cases this shift equals 1 channel number every 3 weeks. After storage until the expiration date, histograms of preserved red blood cell populations become distinctly narrower. Values for the absolute distribution width at half peak height are reduced by $6-17 \%$.

Table 1 shows haemocytometric values. With variations from batch to batch, red blood cell counts decrease by $1-3 \%$ in the first 4 weeks and $4-8 \%$ in the next 3 weeks. Four weeks after receiving the preserved red blood cell suspensions their mean corpuscular volume values are $1-4 \%$ lower. After a total of 7 weeks their values are decreased by $4-8 \%$. Consequently, in the case of haematocrit values, more pronounced shifts are observed: after 4 weeks $2-6 \%$ lower values, reaching $6-12 \%$ after 7 weeks. Especially in the second half of the storage period the values of the haematocrit show progressive changes.

\section{Applications}

In figure 3 a red blood size histogram of a patient with microcytic anaemia due to iron depletion is depicted. In figure 4 a size distribution histogram of a patient with macrocytic anaemia is shown. The haemocytometric values for both subjects are listed in the legends of these figures. The red cell populations reflect the result of the erythropoiesis during the past 4 months. In the case of iron deficiency the size distribution histogram shows a marked shift to the left. The mean corpuscular volume is $70 \mathrm{fl}$. The absolute distribution width at half peak height is increased, reflecting a markedly increased heterogeneity in the red cell population. 
Tab. 1. Effects of ageing on different batches of preserved Ortho Abnormal and Normal Blood Controls.

Mean values are determined from measurements during the $1^{\text {st }}$ week after receipt. Percentage deviation for the haemocytometric values was estimated after 4 and 7 weeks, respectively, from the date of receipt.

\begin{tabular}{|c|c|c|c|}
\hline \multicolumn{2}{|c|}{$\begin{array}{l}\text { Ortho } \\
\text { "abnormal" blood control }\end{array}$} & \multicolumn{2}{|c|}{$\begin{array}{l}\text { Ortho } \\
\text { "normal" blood control }\end{array}$} \\
\hline $\begin{array}{l}\text { Mean } \\
\text { value }\end{array}$ & $\begin{array}{l}\text { Percentage } \\
\text { deviation after }\end{array}$ & $\begin{array}{l}\text { Mean } \\
\text { value }\end{array}$ & $\begin{array}{l}\text { Percentage } \\
\text { deviation after }\end{array}$ \\
\hline 1st week & 4 weeks 7 weeks & 1st week & 4 weeks 7 weeks \\
\hline
\end{tabular}

Red blood cell concentration $\left[10^{12} / 1\right]$

$\begin{array}{llllll}2.00 & -2 & -5 & 3.78 & -1 & -3 \\ 2.35 & -2 & -4 & 4.01 & -2 & -5 \\ 2.30 & -3 & -8 & 4.10 & -1 & -6 \\ 2.40 & -2 & -5 & 3.90 & -1 & -5 \\ 2.27 & -3 & -5 & 4.10 & -2 & -4\end{array}$

Haematocrit

$\begin{array}{lrrrrr}0.188 & -4 & -12 & 0.354 & -2 & -6 \\ 0.220 & -3 & -10 & 0.382 & -3 & -8 \\ 0.219 & -5 & -12 & 0.410 & -5 & -10 \\ 0.230 & -5 & -9 & 0.366 & -6 & -9 \\ 0.204 & -2 & -10 & 0.380 & -4 & -6\end{array}$

Mean corpuscular volume [fl]

$\begin{array}{lllrll}94 & -4 & -7 & 94 & -2 & -4 \\ 93 & -1 & -6 & 95 & -2 & -4 \\ 95 & -3 & -6 & 100 & -3 & -6 \\ 94 & -4 & -8 & 93 & -3 & -6 \\ 90 & -2 & -6 & 92 & -1 & -4\end{array}$

Absolute distribution width*

$\begin{array}{lrrrrr}38 & -4 & -6 & 39 & -11 & -15 \\ 41 & -10 & -16 & 39 & -13 & -17 \\ 40 & -5 & -11 & 40 & -12 & -16 \\ 39 & -8 & -12 & 35 & -10 & -13 \\ 38 & -5 & -8 & 38 & -14 & -17\end{array}$

Light scattering intensity peak**

$\begin{array}{rrrrrr}30 & -3 & -7 & 30 & -6 & -13 \\ 30 & -3 & -7 & 31 & -3 & -6 \\ 30 & -3 & -7 & 33 & -3 & -6 \\ 31 & -6 & -13 & 30 & -3 & -6 \\ 30 & -3 & -7 & 30 & -3 & -6\end{array}$

* absolute distribution width of a red cell size histogram at half maximum (femtoliter)

** light scattering intensity, expressed as voltage channel number derived from the red cell size distribution hịstogram.

In contrast, in vitamin $B_{12}$ deficiency (figure 4), a histogram is depicted indicating the presence of macrocytic red cells together with the remaining normalsized red cells. The size dispersion of the red cells is obviously increased.

\section{Discussion}

Several methods are used for the preservation of human red blood cells. Commercial interest and nonelucidated problems encountered in blood preserva-

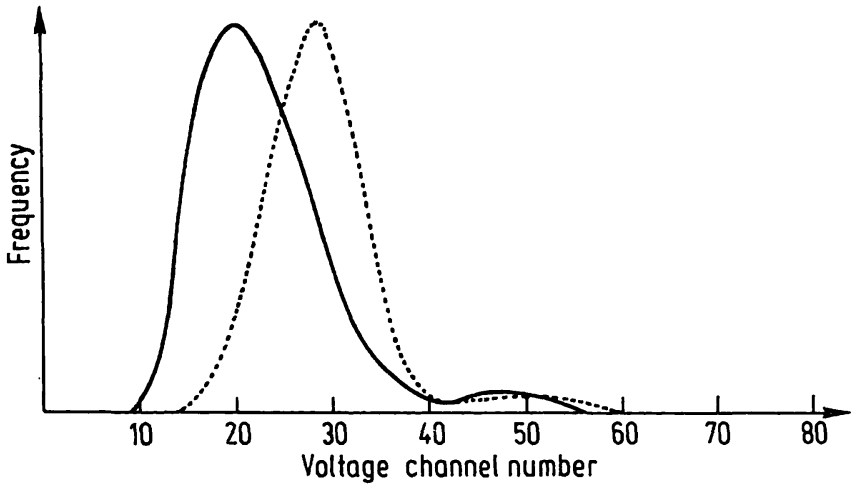

Fig. 3. Red cell size distribution histogram from a female subject with anaemia due to iron depletion. The dotted line represents a reference distribution curve.

Haemocytometric values:

Red cell concentration

Haematocrit

$3.43 \cdot 10^{12} / 1$

Mean corpuscular volume

$0.2401 / 1$

Absolute distribution width at half $45 \mathrm{fl}$ peak beight

Histogram peak value (expressed as 20 voltage channel number)

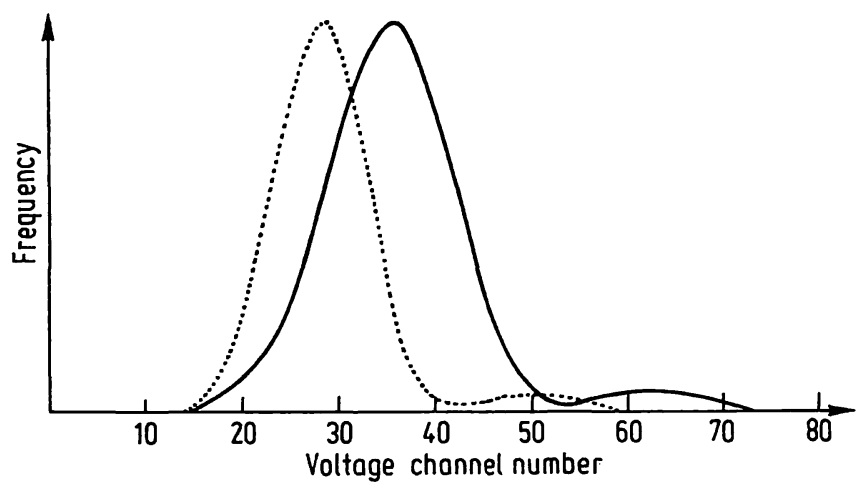

Fig. 4. Red cell size distribution histogram from a male subject with pernicious anaemia. The dotted line represents a reference size distribution curve.

Haemocytometric values:

Red cell concentration

Haematocrit

Mean corpuscular volume

$3.04 \cdot 10^{12} / 1$

Absolute distribution width at half

$118 \mathrm{fl}$

peak height

Histogram peak value (expressed as voltage channel number)

$51 \mathrm{fl}$

36

tion are amongst the reasons for the scarcity of literature on this subject. Therefore more quantitative information is needed concerning the degree of deterioration of red cells during storage of preserved blood control specimens.

Membrane characteristics of red blood cells are more or less influenced by the method of preservation. Aldehyde-fixed red blood cells are stable over a very long period of time. However, they have some disadvantages such as a tendency to clump and to adhere to walls of the containers and inner surfaces of flow 
channels (4). In fact, these undiluted fixed red blood cell specimens are not suitable for fully automated counters (6). Moreover, the rigidity of the membranes caused by the rigorous pretreatment makes them quite different from native red blood cells. Alternatively, milder preservation methods yielding red cells more similar to untreated cells may be employed. According to specifications supplied by Ortho their blood controls should have constant haemoglobin and red blood cell concentrations until the expiration date, although oxidation and haemolysis gradually increase (7). When the oxidant stress exceeds the normal reducing power, red cell damage may occur, because the red cell membrane is susceptible to oxidation of the lipid-protein bilayer. In the course of time, denaturation and precipitation of haemoglobin in the red cell may gradually occur, if the normal protection mechanisms against oxidants fail to maintain haemoglobin in the reduced state. Other proteins may also be affected, resulting in loss of viability and destruction of the red cells (8).

In a Coulter S plus II Hematology Analyzer ghosts formed due to lysis of red blood cells give rise to mean corpuscular volume values in the range from $50-70 \mathrm{fl}$ (4). Particles in this size range are still classified as red blood cells. This explains why red cell counts in blood controls seem to be constant even for several months, when measurements are based on the aperture-impedance principle, although lysis gradually proceeds.

In contrast, measurements based on light scattering intensity show that red blood cell parameters are less stable, and expiration periods must be shortened accordingly. Table 1 shows haemocytometric values measured soon after preservation of the blood specimens and the relative deviations after 4 and 7 weeks from the date of receipt. After storage of blood controls, their red cell histograms become considerably narrower and consequently the peaks reach higher levels. The whole histogram shifts to the left.

Our explanations for these phenomena are purely hypothetical. The decrease in light scattering intensity may be due to changes in red cell surface characteristics and the surface area. Loss of membrane material in the form of microvesicles has been noted during storage of normal red cells (9). The tendency to vesiculate may be a generalized characteristic of the stressed red cell membrane. The phenomenon of vesiculation is accompanied by a slight decrease of the water content, resulting in some reduction of the red cell volume. When vesiculation proceeds, red cells gradually progress from discocytes to echinocytes (10). Echinocytes are probably formed during storage for some weeks. In stored blood samples, increasing numbers of echinocytes may be present due to ATP depletion and lysolecithin formation in the plasma (11). In some blood control batches remnants of red cells develop; they are illustrated by a growing subpopulation on the lower left limb of the histogram.

The manufacturer's instructions for handling blood control specimens should be considered very carefully to reduce deterioration as much as possible. Repeated heating and cooling cycles should be avoided. Bacterial contamination gives rise to inaccurate results: after sampling vials should be tightly sealed immediately. Opened vials may not be used more than 7 consecutive days provided no contamination has already occurred. Contamination of a specimen is sometimes evidenced by darkening of the red cells, and haemolysis.

We have tested several other specimens of commercially available blood controls, e. g. Para 8 (12), Instrublood (13). With respect to our criteria, all of these specimens to a greater or lesser degree, suffer from a lack of sufficient stability in time. However, using the ELT 800 the latter blood controls frequently caused trouble, because the sample was aspirated too slowly, sometimes clogging the measurement system.

For this reason, in our laboratory, monodisperse polystyrene spheres are preferred for assuring a more reliable calibration of the haematology analyser. No distinct limitations are known with respect to expiration intervals for Dynosheres ${ }^{\circledR}$. The suspensions show perfect stability with time. Size distribution histegrams confirm that the particles are rather monodisperse; coefficients of variation as experimentally determined from their size distributions are in agreement with the specifications supplied by the manufacturer. In the calibration procedure errors caused by incorrect optical alignment, accumulation of cell debris and proteins in the flow cell, or errors in phasing or the setting of discrimination tresholds etc. can therefore be detected. Artificial reference samples guarantee optimal reliability over extended periods. Preserved blood controls more closely resemble the physical properties of anticoagulated human whole blood. After primary calibration of the instrument by use of polystyrene spheres the application of blood cell controls with medium term stability remains necessary to check the performance of the instrument and the reagents. Due to deterioration of red blood cells with time optimal performance of measuring red cell characteristics and the generation of size histograms cannot be guaranteed by applying only preserved blood controls. 
Characteristic pictures from two subjects with iron depletion and vitamin $B_{12}$ deficiency respectively, are demonstrated in figure 3 and 4 . Both histograms reveal increased heterogeneity in their red cell sizes. In nutritional deficiency, the peripheral blood may show a conglomerate of successively produced red cell

\section{References}

1. Lombarts, A. J. P. F. (1985) Studies on quality assurance in hemocytometry, Thesis Erasmus University, Rotterdam, The Netherlands.

2. Bull, B. S. (1984) In: Laboratory Hematology (Koepke, J. A., ed.) pp. 1003-1004, Churchill Livingstone, New York.

3. Lombarts, A. J. P. F. \& Leynse, B. (1984) Clin. Chim. Acta. $139,145-154$.

4. Lombarts, A. J. P. F. \& Leynse, B. (1983) Ann. Clin. Biochem. 20, 302-307.

5. Operator Reference Manual Ortho ELT 800 Hematology Analyzer (laser based), part no 168-0010-005. Ortho Diagnostic Systems Inc, Westwood, MA 02090.

6. Cavill, J. (ed.) (1982) Methods in hematology, Vol 4: Quality control Churchill Livingstone, New York.

7. Package Insert Ortho Hematology Control Blood, Revision December 1984, code 01122/3. Ortho Diagnostic Systems n. v., B 2340 Beerse, Belgium. populations with markedly different cell sizes (14). Microcytic or macrocytic cells will contrast with normal-sized red blood cells. If the Hematology Analyzer is perfectly adjusted, red cell size histograms from these subjects can be used for assessing the adequacy of substitution therapy after some weeks.

8. Koepke, J. A. (1984) In: Laboratory Hematology (Koepke, J. A., ed.), p. 492, Chirchill Livingstone, New York.

9. Rumsby, M. G., Trotter, J., Allan, D. \& Mitchell, R. H. (1977) Biochem. Soc. Trans. 5, 126-128.

10. Wagner, G. M., Chiu, T. Y., Yee, M. C. \& Lubin, B. H. (1986) J. Lab. Clin. Med. 108, 315-324.

11. Van Assendelft, O. W. (1984) In: Laboratory Hematology (Koepke, J. A., ed.) p. 885, Churchill Livingstone, New York.

12. Package Insert Para 8 Hematology Reference Control, Form 3083-2 (May 1986). Streck Laboratories, Ind., 14306 Industrial Road, Omaha, NE 68137.

13. Lombarts, A. J. P. F. \& Leynse, B. (1984) Clin. Chim. Acta. 143, 7-15.

14. Bessman, J. D. (1980) Johns Hopkins Med. J. 146, $226-$ 230.
P. C. Bartels, Department of Clinical Chemistry and Haematology Medical Center Alkmaar Van Everdingenstraat 18 NL-1814 HA Alkmaar 
